\title{
SAR TRAFFIC MONITORING USING TIME-FREQUENCY ANALYSIS FOR DETECTION AND PARAMETER ESTIMATION
}

\author{
Stefan V. Baumgartner, Gerhard Krieger \\ Microwaves and Radar Institute, German Aerospace Center (DLR), 82234 Wessling, GERMANY \\ Email: stefan.baumgartner@dlr.de
}

\begin{abstract}
In the paper a ground moving target indication (GMTI) algorithm operating on preprocessed range-compressed SAR data is presented. For preprocessing range cell migration correction for stationary targets in most practical cases is sufficient. Moving target signal detection and extraction, adaptive range cell migration correction, position and across- as well as along-track velocity estimation is then performed without a priori knowledge by using matched filter banks and the fractional Fourier transform. The proposed algorithm is able to cope with multi-component linear frequency modulated signals as they arise in real traffic scenarios containing a number of moving road vehicles.
\end{abstract}

Index Terms-moving target indication (MTI), motion parameter estimation, synthetic aperture radar, fractional Fourier transform

\section{INTRODUCTION}

The large number of moving vehicles in real road traffic scenarios and additionally the possibly small spacing between them implicate that raw or range-compressed data based algorithms have to cope with overlapping or crossing range histories of moving target signals. Separating these signals from each other in the range-compressed data domain (e.g. by tracking the range history or signal energy, respectively [1]) for detection, motion parameter estimation and imaging purposes is still challenging, especially under low SNR conditions and strong target scintillation. One way out is the use of preprocessed range-compressed data where the major part of the range cell migration of the moving target signal already has been corrected. Instead of tracking range histories of particular targets now azimuth lines (= lines of constant range) are picked out from the clutter suppressed data matrix successively for further analysis. Using dual- or multi-channel SAR systems clutter suppression simply can be done by applying for example the DPCA technique [2] (cf. Fig. 1). Each of the picked out azimuth lines may now contain multi-component linear frequency modulated (LFM) moving target signals. For detecting these LFM signals several techniques are known in the literature, for example the Wigner-Hough transform, the matched filer bank (MFB) principle and also the fractional Fourier transform (FrFT) [3].

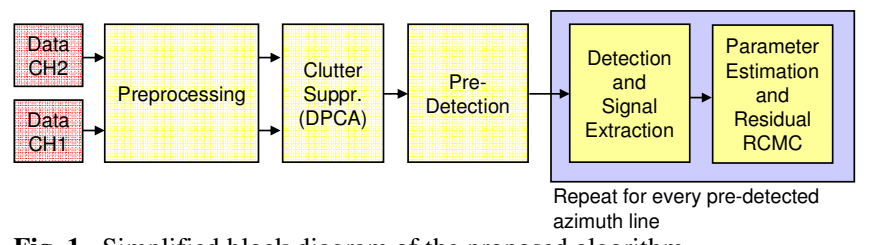

Fig. 1. Simplified block diagram of the proposed algorithm.

In the following the proposed GMTI algorithm is explained. First the considered moving target motion equations and the corresponding range history are presented. Afterwards the residual range migration for moving targets is derived. The knowledge of the residual range migration is essential for the parameter estimation steps. It is shown how residual range migration can principally be compensated for each target adaptively. Then the FrFT and the MFB are compared for detection purposes. Finally, the parameter estimation steps are presented and discussed before the paper is concluded.

\section{MOVING TARGET RANGE HISTORY}

It is assumed that the target moves on ground plane $(z=0)$ with constant acceleration during the observation time. The motion equations can then be written as

$$
x(t)=x_{0}+v_{x 0} t+\frac{1}{2} a_{x} t^{2}, \quad y(t)=y_{0}+v_{y 0} t+\frac{1}{2} a_{y} t^{2},
$$

where $a_{x}$ and $a_{y}$ are the constant acceleration components in along-track and across-track direction and $v_{x 0}$ and $v_{y 0}$ are the velocity components at beam center time $t=0$. The alongtrack position of the target at $t=0$ is denoted as $x_{0}$ and the across-track position as $y_{0}$. The distance from the transmit antenna to the target is given as

$$
r(t)=\sqrt{\left(x(t)-v_{p} t\right)^{2}+y(t)^{2}+h^{2}},
$$

where $h$ is the altitude of the radar platform above ground and $v_{p}$ is the along-track velocity of the platform. After 
performing a second order Taylor approximation and some substitutions above equation can be written as

$$
r(t) \cong r_{0}-\frac{\lambda}{2} f_{D C} t-\frac{\lambda}{4} k_{a} t^{2}
$$

where $r_{0}$ is the distance between the transmit antenna and the target at $t=0, \lambda$ is the radar wavelength, $f_{D C}$ is the Doppler shift and $k_{a}$ the Doppler slope of the received signal given as

$$
\begin{gathered}
f_{D C}=-\frac{2}{\lambda r_{0}}\left[x_{0}\left(v_{x 0}-v_{p}\right)+y_{0} v_{y 0}\right], \\
k_{a}=-\frac{2}{\lambda r_{0}}\left\{x_{0} a_{x 0}+\left(v_{x 0}-v_{p}\right)^{2}+y_{0} a_{y 0}+v_{y 0}^{2} .\right. \\
\left.-\frac{1}{r_{0}^{2}}\left[x_{0}\left(v_{x 0}-v_{p}\right)+y_{0} v_{y 0}\right]^{2}\right\}
\end{gathered}
$$

Substituting $t=\left(f_{a}-f_{D C}\right) / k_{a}$ into (3) the quadratic approximation of the range history as a function of Doppler frequency $f_{a}$ is obtained:

$$
r\left(f_{a}\right)=r_{0}+\frac{\lambda f_{D C}^{2}}{4 k_{a}}-\frac{\lambda}{4 k_{a}} f_{a}^{2} .
$$

\section{RESIDUAL RANGE MIGRATION CORRECTION}

After performing common range cell migration correction (RCMC) for stationary targets, which for example simply can be done by just omitting the azimuth focusing stage of a common chirp scaling SAR processor [4], the residual range migration of the moving target signal is given as

$$
\Delta r\left(f_{a}\right)=\frac{\lambda}{4}\left(\frac{f_{D C}{ }^{2}}{k_{a}}-\frac{f_{D C, S T}{ }^{2}}{k_{a, S T}}\right)+\frac{\lambda}{4}\left(\frac{1}{k_{a, S T}}-\frac{1}{k_{a}}\right) f_{a}^{2},
$$

where $f_{D C, S T}$ and $k_{a, S T}$ are the Doppler shift and Doppler slope of a stationary target located at $t=0$ at the same position as the moving target:

$$
f_{D C, S T}=\frac{2 x_{0}}{\lambda r_{0}} v_{p}, \quad k_{a, S T}=-\frac{2}{\lambda r_{0}}\left(1-\frac{x_{0}^{2}}{r_{0}^{2}}\right) v_{p}{ }^{2} .
$$

From equation (7) it can be seen that the moving target signal after "stationary" RCMC is displaced in range (first term; in the airborne case the range displacement may be in the order of dozens of meters) and that the residual range migration has quadratic behavior (second term). In Fig. 2 simulated range-compressed signals with different motion parameters after RCMC for stationary targets are shown in the range-azimuth domain (time domain) where the effect of the residual range cell migration clearly can be recognized.

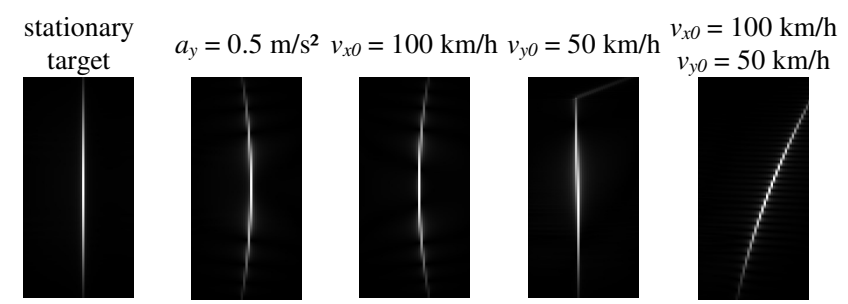

Fig. 2. Simulated range compressed moving target signals after RCMC adapted for stationary targets (horizontal axis: range; vertical: azimuth).
For simulation an X-band airborne system with $v_{p}=90 \mathrm{~m} / \mathrm{s}$, $h=2200 \mathrm{~m}, r_{0}=3810 \mathrm{~m}, \lambda=0.0312 \mathrm{~m}$, pulse repetition frequency $P R F=2500 \mathrm{~Hz}$ and range bandwidth of $100 \mathrm{MHz}$ was used. The quadratic part of the residual range migration is mainly determined by the Doppler slope $k_{a}$ which itself depends strongly on the across-track acceleration $a_{y}$ and the along-track velocity $v_{x 0}$ as can be seen from Equ. (5).

Assuming that $k_{a, S T}$ is known and $k_{a}$ can be estimated prior to residual RCMC using a MFB or FrFT (cf. section 4), the residual quadratic range migration can then be corrected by performing a Doppler frequency dependent range shift of the image patch containing the target (cf. section 5):

$$
\Delta r_{\text {quadratic }}\left(f_{a}\right)=-\frac{\lambda}{4}\left(\frac{1}{k_{a, S T}}-\frac{1}{k_{a}}\right) f_{a}^{2} .
$$

This range shift can for example be done in the wavenumber domain by multiplying linear phase ramps. In Fig. 3 the range-compressed signals after performing residual RCMC are shown. The major part of the signal energy is now concentrated along azimuth, i.e. in a single azimuth line.

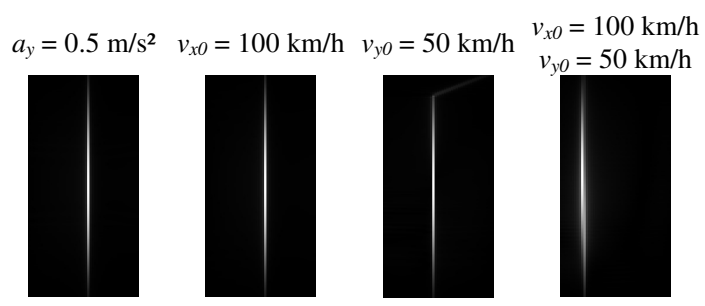

Fig. 3. Simulated range compressed moving target signals after residual RCMC (horizontal axis: range; vertical: azimuth).

\section{DETECTION AND SIGNAL EXTRACTION}

Detection is done by successively picking out azimuth lines and transforming them into the fractional Fourier domain using different assumptions of the rotation angle $\alpha$ which is related to the Doppler slope in the following way [5]:

$$
\alpha=\operatorname{atan}\left(-\frac{1}{k_{a}} \frac{P R F^{2}}{N}\right),
$$

where $N$ is the number of samples contained in the azimuth line. By applying different rotation angles a "Fractional Fourier Spectra Map" is obtained (cf. Fig. 4, left). Moving target detection is now reduced to peak detection [10]. Each peak corresponds to a moving target, a certain Doppler slope $k_{a}$ and a "displaced azimuth position".

Every detected moving target signal can be extracted and separated from the others by performing bandpass filtering in the fractional Fourier domain (cf. Fig. 4, right). The computational time for detection can be decreased by using a pre-detector (cf. Fig. 1) such that only the azimuth lines containing possible moving target candidates are chosen for the investigations. 


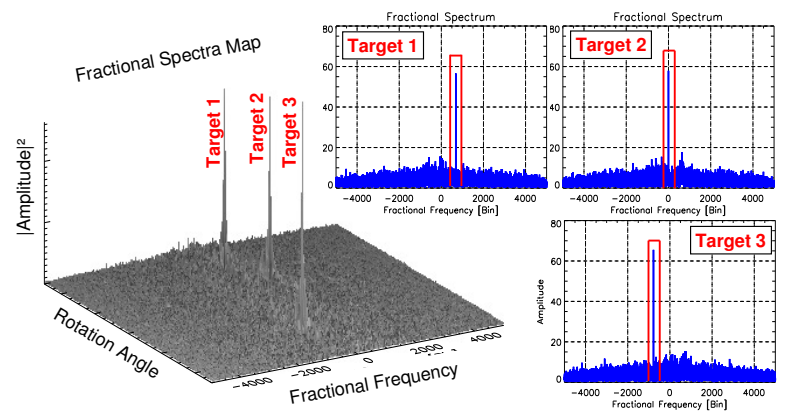

Fig. 4. Fractional spectra map containing three simulated moving targets (left) and the corresponding fractional spectra for each target (right). The bandpass filter windows to apply for signal extraction are sketched in red.

Furthermore, instead of using the time consuming FrFT also a quite faster matched filter bank can be used for detection purposes. The matched filter in Doppler domain is given as

$$
S_{\text {filter }}\left(f_{a}\right) \cong \exp \left(-j \pi \frac{1}{k_{a}} f_{a}^{2}\right),
$$

and the focused signal in time domain is then obtained by

$$
I(t)=F^{-1}\left\{S\left(f_{a}\right) S_{\text {filter }}^{*}\left(f_{a}\right)\right\},
$$

where $S\left(f_{a}\right)$ is the azimuth line transformed to Doppler domain and $F^{-1}$ is the inverse Fourier transform. After performing processing time comparisons on a common personal computer with a Intel dual core $2.66 \mathrm{GHz}$ processor and 2 GB RAM, we found that the matched filter is almost 20 times faster than the FrFT (for comparison we have used our own IDL [6] implementation of the FrFT which is based on the fast approximation of the continuous FrFT [7] and a free available MATLAB realization [8]). This processing time difference implies that for time critical applications the MFB should be used for detection and afterwards the FrFT only for signal extraction.

The detected peak position $N_{\text {img, } M F}$ of a moving target signal in the MFB is related to the peak position $N_{i m g, F r}$ of the same signal in the fractional Fourier domain (cf. Fig. 5, left) in the following way (linear time-frequency history assumed):

$$
N_{i m g, F r}=\left(N_{i m g, M F}+M \cdot N-\frac{N}{2}\right) \cos \alpha+\frac{N}{2},
$$

where $M$ is an integer number considering possible backfolding of the focused signal along azimuth time after matched filtering (cf. Fig. 5 right).
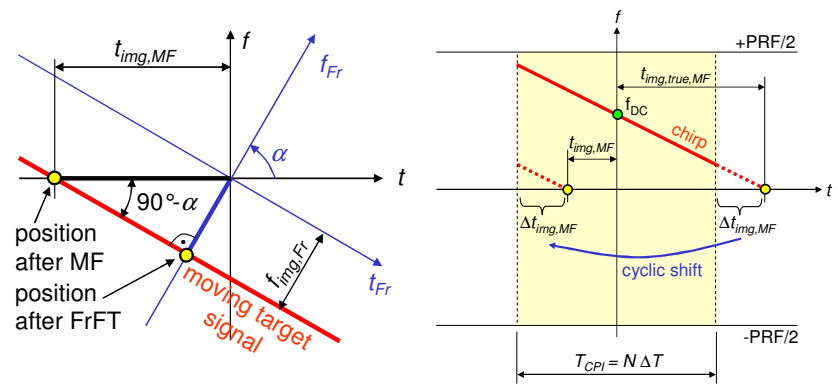

Fig 5. Time-frequency representations of a moving target signal (left: comparison with FrFT; right: backfolding due to cyclic shift).
Such backfolding may occur, if the observation time of the picked out azimuth line is not larger than the synthetic aperture time. The ambiguity of $M$ can be resolved by looking at all expected peak positions and finally choosing that peak where the signal has highest amplitude. Also several neighboring samples around the expected positions should be considered if real SAR data is used, since higher order phase terms and a non-linear time-frequency history may cause a residual position error between the peaks.

In many practical cases it is not necessary to perform residual RCMC prior to detection. In Fig. 6 (right) the response of a MFB is shown. As input a single DPCA clutter suppressed azimuth line containing several targets with overlapping range histories has been used (cf. Fig. 6, left). The data were acquired with DLR's E-SAR system in Germany in the Chiemsee region in the year 2005.

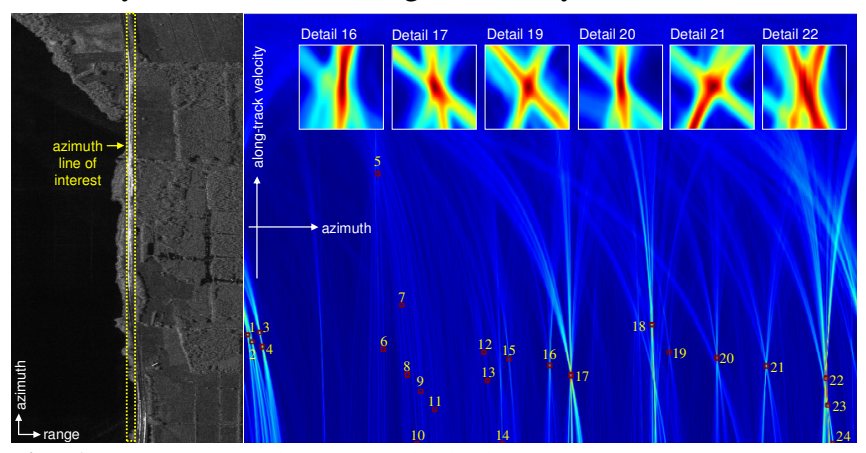

Fig. 6. X-band SAR image acquired with DLR's two-channel aperture switching E-SAR system (left) and matched filter bank response of the picked out and clutter suppressed azimuth line of interest (right).

All targets have moved with high velocities between 80 and $140 \mathrm{~km} / \mathrm{h}$ only in along-track direction. A simple peak detector was applied without comparing the SCNR of the peaks to a threshold. Therefore the peaks numbered with 5 to 15 and 24 are possibly false detections. The detection of these signals having no across-track velocity components was probably possible due to high target velocity and large processed azimuth bandwidth so that a certain prominent scatterer moves trough more than only one azimuth resolution cell between two different observations from the same point in space. A squinted acquisition geometry also introduces a small line-of-sight velocity component and hence an along-track interferometric (ATI) phase.

If the residual range migration prior to detection is severe so that an accurate estimation of $k_{a}$ might be not possible (cf. Fig. 2, right), also different assumptions of the across-track velocity can be incorporated into the preprocessing step and additionally the range resolution can be increased.

\section{PARAMETER ESTIMATION}

Due to the fact that "stationary" RCMC is performed as a pre-processing step, all moving targets will be displaced in range. In case of $x_{0}<<r_{0}$ (i.e. negligible small squint angles 
and centered target tracks so that $x_{0}$ can be assumed to be zero) Equ. (7) can be written as

$$
\Delta r\left(f_{a}\right)=\frac{v_{r 0}{ }^{2}}{\lambda k_{a}}+\frac{\lambda}{4}\left(-\frac{\lambda r_{0}}{2 v_{p}{ }^{2}}-\frac{1}{k_{a}}\right) f_{a}^{2} .
$$

Even if $r_{0}$ up to now is not known accurately and instead the range where the target was detected is inserted into above equation, the quadratic part of the residual range cell migration can roughly be corrected so that most of the target energy is then distributed along one single azimuth line (cf. section 3). Also the "new" range of this azimuth line after residual RCMC can be estimated relative to the range where the signal was detected (remember that for detection the azimuth line with residual range cell migration was used so that it is not known which part of the signal has been detected). For estimating the "new" range of the RCMC azimuth line, the Doppler frequency band and thus, the corresponding $f_{a}$ of the detected signal prior to residual RCMC has to be estimated (cf. Fig. 7). This estimation simply can be done by transforming the detected and extracted moving target signal into Doppler domain and performing a Doppler centroid estimation.

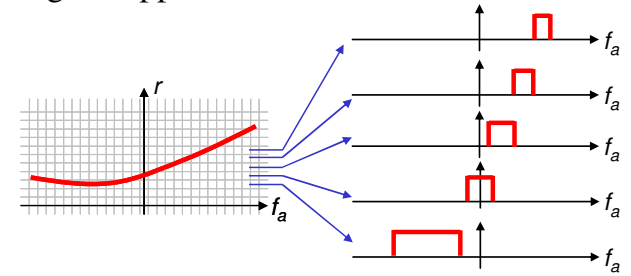

Fig. 7. Residual range migration (left) and corresponding range dependent Doppler spectra.

Before extracting the major part of the target energy from the RCMC azimuth line located at the "new" range it is recommended to refine the estimation of $k_{a}$ using again a "Fractional Fourier Map" but now considering only $k_{a}$ values around the already known "old" $k_{a}$ estimate. The bandpass filter window for extraction has to be large enough so that also parts of the signal deviating from the linear timefrequency history are not filtered out. Especially at high range resolution SAR systems the range bandwidth should be reduced (i.e. increase of range resolution) prior to any detection and parameter estimation steps so that also signal parts having higher order residual range migration are located in one single azimuth line after correction of the residual quadratic range cell migration.

The line-of-sight velocity $v_{r 0}$ can be range independent estimated using for example the fractional ATI phase [5]. Together with $k_{a}$ the range $r_{0}$ can be computed with high accuracy as well as the beam center across-track position, the along-track velocity and even the across-track acceleration of the moving target [9]. Fig. (8) shows the results obtained from the proposed algorithm applied on real multi-channel data acquired with DLR's new F-SAR system in 2007. Three moving targets have been detected and their parameters have been estimated automatically (for details regarding parameter estimation cf. [9]).

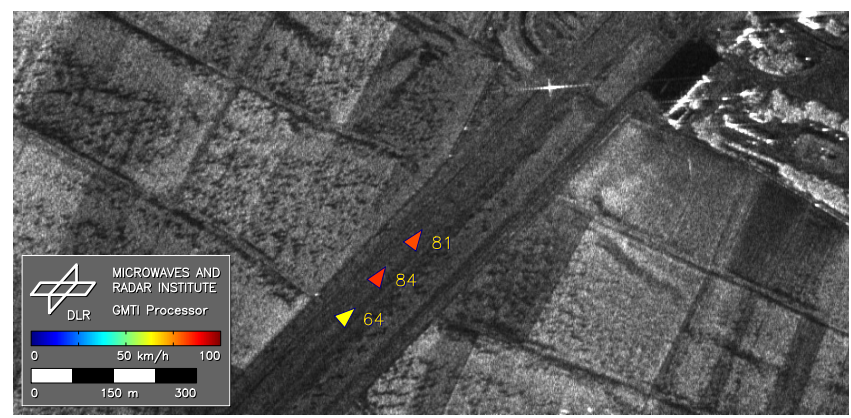

Fig. 8. SAR image acquired with DLR's new X-band four-channel F-SAR system containing three detected moving targets. Detection was done using the proposed algorithm (horizontal axis: range; vertical axis: azimuth).

\section{CONCLUSIONS}

In the paper an algorithm for ground moving target indication, signal extraction and parameter estimation operating on preprocessed range-compressed data was presented. Due to preprocessing the moving target signal energy is displaced in range and special attention has to be taken for estimating the true range position. The applicability of the proposed algorithm was verified using simulated and real SAR data.

\section{REFERENCES}

[1] C.H. Gierull and I. Sikaneta, "Raw Data based Two-Aperture SAR Ground Moving Target Target Indication," Proceedings of IGARSS 2003, Toulouse, France, July 2003.

[2] F.R. Dickey and M.M. Sante, "Final Report on Anti-Clutter Techniques," General Electric Co., Heavy Military Elecron. Dept., Rep. No. R65EMH37, Syracuse, NY, 1953.

[3] L.B. Almeida, "The fractional Fourier transform and time-frequency representations," IEEE Transactions on Signal Processing, vol. 42, no. 11, pp. 3084-3091, 1994.

[4] A. Moreira, J. Mittermayer and R. Scheiber, "Extended Chirp Scaling Algorithm for Air- and Spaceborne SAR data processing in Stripmap and ScanSAR Imaging Modes," IEEE Transactions on Geoscience and Remote Sensing, vol. 34, no. 5, pp. 1123-1136, Sept. 1996.

[5] S. Chiu, "Application of Fractional Fourier Transform to Moving Target Indication via Along-Track Interferometry,” EURASIP Journal on Applied Signal Processing, vol. 20, pp. 3293-3303, 2005.

[6] "IDL: Interactive Data Language," June 2008, [Online]. Available: http://rsinc.com/idl/.

[7] H.M. Ozaktas, O. Arikan, M.A. Kutay and G. Bozdagi, "Digital Computation of the Fractional Fourier Transform," IEEE Transactions on Signal Processing, vol. 44, no. 9 , pp. 2141-2150, Sept. 1996.

[8] J. O'Neill, "Discrete TFDs: A Collection of Matlab files for TimeFrequency Analysis," June 2008, [Online]. Available: http://www.mathworks.com/matlabcentral/fileexchange/loadFile.do?ob jectId=63\&object Type $=$ file.

[9] S.V. Baumgartner and G. Krieger, "Acceleration Independent AlongTrack Velocity Estimation of Moving Targets," Proceedings of EUSAR 2008, Friedrichshafen, Germany, June 2008.

[10]H.-B. Sun, G.S. Liu, H. Gu and W.-M. Su, "Application of the Fractional Fourier Transform to Moving Target Detection in Airborne SAR," IEEE Transactions on Aerospace and Electronic Systems, vol. 38 , no. 4, pp. 1416-1424, October 2002. 